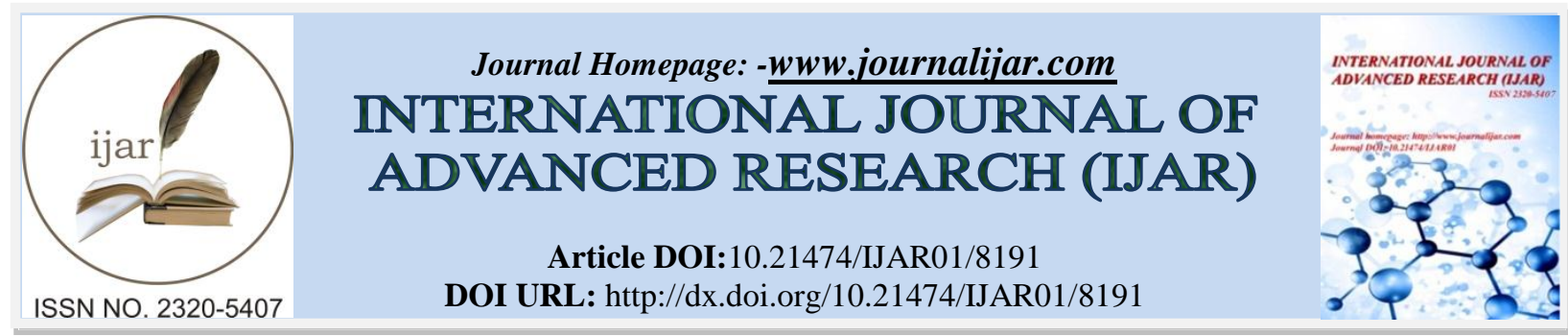

RESEARCH ARTICLE

\title{
EFFECT OF TAURINE ON DEPRESSION ASSOCIATED WITH OBESITY AND STREPTOZOTOCIN INDUCED DIABETES IN RATS.
}

\author{
Maram Mohamed El Tabaa, Nahid Mohamed El Fateh Tahoon, Mahmoud Abd El Hamied El Gharib and \\ Mohamed Mohamed Mady. \\ Department of Medical Physiology, Faculty of Medicine, Tanta University, Egypt.
}

\section{Manuscript Info}

\section{Manuscript History}

Received: 10 October 2018

Final Accepted: 12 November 2018

Published: December 2018

Key words:-

Taurine, Diabetes mellitus, Obesity, Depression, BDNF, Serotonin.

\section{Abstract}

Aim of the Work: to investigate the effect of taurine on depression associated with obesity and streptozotocin induced diabetes in rats. Methods: 60 male rats were divided into six groups (10 rats each): (I:Control group) received saline intraperitoneally once a day for 30 days, (II: Taurine group) received taurine $(100 \mathrm{mg} / \mathrm{kg})$ intraperitoneally once a day for 30 days, (III: Diabetic group) received a single dose of streptozotocin $(50 \mathrm{mg} / \mathrm{kg}$ ) intraperitonealy, (IV: Obese group) were fed with high fatty diet for 8 weeks, (V:Taurine treated diabetic group) received taurine $(100 \mathrm{mg} / \mathrm{kg})$ intraperitoneally once a day for 30 days after induction of diabetes, (VI: Taurine treated obese group) received taurine $(100 \mathrm{mg} / \mathrm{kg})$ intraperitoneally once a day for 30 days after induction of obesity.

Results:Taurine treated groups showed significant decrease in serum glucose, brain malondialdehyede (MDA), tumor necrosis factor alpha (TNf- $\alpha$ ) and immobility time of forced swim test with significant increase in brain catalase, brain derived neurotropic factor (BDNF), serotonin and numbers of squares crossed in open field test. In addition, there is significant increase in serum insulin and body mass index with insignificant changes in insulin resistance and serum adiponectin level in taurine treated diabetic group. However, there is significant decrease in serum insulin, insulin resistance with significant increase in serum adiponectin, but insignificant change in body mass index in taurine treated obese group.

Conclusion: Antidepressent effect of taurine has can be explained by its hypoglycaemic, antioxidant, anti-inflammatory and neurotrophic effects.

Copy Right, IJAR, 2017,. All rights reserved.

\section{Introduction:-}

Diabetes is a serious metabolic disorder with high incidence of various complications affecting physical and emotional well-being which make diabetes a challenging disease for management (Quinn et al., 2017). Presence of co-morbid depression is usually associated with poor management of diabetes due to impaired glycaemic control, high incidence of complications and decreased response to medication (Kearns et al., 2017).

Corresponding Author:-Maram Mohamed El Tabaa.

Address:-Department of Medical Physiology, Faculty of Medicine, Tanta University, Egypt. 
Obesity is a major public health problem and its prevalence is increasing worldwide which in turn increases the burden of its associated co-morbidities as cardiovascular disease, lipid disorders, hypertension, diabetes and mood disorders (Sharma \& Fulton, 2013). However, the relationship between obesity and psychiatric conditions such as major depressive disorder is still unclear (Sweeney et al., 2017)

Major depressive disorder (MDD), known simply as depression, is one of the most prevalent neuropsychiatric disorders associated with high morbidity and mortality (Kim et al., 2016). It has been expected that depression will become the second leading cause of disability worldwide after human immunodeficiency virus (HIV) in 2030 (Ma et al., 2016).

Regarding the pathophysiology of MDD, no established mechanism can explain all aspects of the disease since it is complex and involving numerous neurobiological systems which include dysegulation of neurotrophin signaling pathways(Otte et al., 2016) and impairment in serotonin neurotransmission (Ma et al., 2016). Oxidative stress and inflammation play also an integral role in the depression pathophysiology (Schuch et al., 2016).

Treatment with traditional antidepressant drugs may be associated with weight gain and increasing risk of insulin resistance (Lang \& Borgwardt, 2013). It may also affect the blood glucose control and aggravating the hyperglycemic state in patients with diabetes (Zanoveli et al., 2016).

Therapeutic application of nutraceutical supplements like vitamins, minerals and amino acids is an emerging approach to improves the efficacy of antidepressants as they affect many neurobiological pathways involved in depression (Sarris, 2017).

Taurine (2-aminoethanesulphonic acid) has various important physiological functions in the central nervous system such as antioxidation, anti-inflammation, osmoregulation, neuromodulation and neuroprotection(Menzie et al., 2014).

Taurine deficiency is closely related to the development of depression like behaviour, suggesting a possible beneficial effect of taurine supplementation on depression (Wu et al., 2017).

Taurine was approved by the European Food Safety Authority to have no adverse effects up to $1000 \mathrm{mg} / \mathrm{kg} / \mathrm{day}$ (Salze \& Davis, 2015). Accordingly, taurine supplementation as an adjuvant treatment for depression may be helpful without any adverse effects at a suitable dose(Wu et al., 2017).

\section{Materials and Methods:-}

\section{Reagents and Drugs:-}

Taurine (TAU) and Streptozotocin (STZ) were obtained from Sigma-Aldrich Co., USA. Normal and high fat diets (HFD) constituents were provided by El Gomhoureya For Trading Drugs \& Medical Supplies Co., Egypt. Glucose reagent kit was from BioMed Diagnostics Co., Egypt. Rat insulin ELISA kit was obtained from RayBiotech Co., USA. Rat adiponectin ELISA kit was obtained from Boster Biological Technology Co., USA. Malondialdeyde (MDA) and Catalase (CAT) reagent kits were from Bio-Diagnostics Co., Giza, Egypt. Rat Tumor necrosis factor alpha (TNF- $\alpha$ ) ELISA kit from Shanghai Sunred Biological Technology Co., China and Rat Brain derived neurotropic factor (BDNF) ELISA kit from MyBioSource Co., USA were used. Other chemicals were obtained from local chemical suppliers.

\section{Animals:-}

The handling of all experimental animals was carried out in accordance with the instructions of the ethical committee of Faculty of Medicine code no (31113/08/16), Tanta University. The present work was carried out on 60 adult male albino rats ranging in weight between 180-200 gm. The rats were housed in isolated animal cages (5 in each cage), in a standard animal laboratory room temperature and exposed to alternate cycles of $12 \mathrm{~h}$ light- darkness, had free access to tap water and food all over the period of the work. The animals were acclimatized for two weeks prior to the experiment. 


\section{Experimental design:- \\ Rats were randomly divided into six equal groups of 10 rats:- \\ Group I (Control group):- \\ The animals of this group received saline intraperitoneally once a day for 30 days as vehicle.}

\section{Group II (Taurine treated group):-}

The animals of this group received taurine $(100 \mathrm{mg} / \mathrm{kg})$ intraperitoneally once a day for 30 days(Caletti et al., 2015).

Group III (STZ-diabetic group):-

Diabetes was induced by giving a single dose of streptozotocin $(50 \mathrm{mg} / \mathrm{kg}$ ) by intraperitoneal injection ( Morais et al., 2014 ) after dissolving it in $0.1 \mathrm{M}$ sodium citrate buffer ( $\mathrm{pH} 3.0$ ). After 3 days, rats with fasting glucose levels $\geq$ $200 \mathrm{mg} / \mathrm{dl}$ were considered diabetic.

\section{Group IV (Obese Group):-}

Obesity was induced by feeding rats with high fatty (HF) diet (60\% energy from fat) for 8 weeks (Papazoglou et al., 2015).

\section{Group V (Taurine treated diabetic group):-}

After induction of diabetes as in group III, rats received taurine $(100 \mathrm{mg} / \mathrm{kg})$ intraperitoneally once a day for 30 days (Caletti et al., 2015).

Group VI (Taurine treated obese group):-

After induction of obesity as in group IV, rats received taurine $(100 \mathrm{mg} / \mathrm{kg})$ intraperitoneally once a day for $30 \mathrm{days}$ (Caletti et al., 2015).

\section{At the end of the experimental period:-}

All rats were subjected to measurement of their body weights and lengths (nose to anus length) for calculation of Body mass index (BMI) according to (Novelli et al., 2007). Behavioral tests have also been carried out including:

\section{Open field test (OFT):-}

It is used to assay the general and locomotor activity associated with depression in rats. It was carried out according to (Ramos et al., 1997; Patke et al., 2015;Redivo et al., 2016). It consists of a square box, $60 \mathrm{~cm} \mathrm{X} 60 \mathrm{~cm}$ with $30 \mathrm{~cm}$ high opaque walls. Its floor was divided into nine smaller squares of equal dimensions $(20 \mathrm{~cm} \mathrm{X} 20 \mathrm{~cm})$. The behavior was video recorded during $5 \mathrm{~min}$ and number of squares crossed with all four paws was considered a measure of the locomotor activity.

\section{Forced swim test (FST):-}

It is the most widely used model for assessing the depressive-like behaviour in rats. It was carried out according to the method of (Porsolt et al., 1978). Rats were placed in a transparent cylinder $(55 \mathrm{~cm}$ tall, $15 \mathrm{~cm}$ in diameter filled with tap water $\left(24 \pm 1^{\circ} \mathrm{C}\right)$ at a height of $\left.30 \mathrm{~cm}\right)$ to ensure that animals could not touch the bottom of the container with their hind paws or their tails. The animals were forced to swim for a 15 min period (pre-test) and $24 \mathrm{~h} \mathrm{later}$ were subjected to a 5-min test session. A rat was judged to be immobile whenever it remained floating in the water, in an upright position, making only small movements to keep its head above water. The time period (in seconds) during which rats were immobile was video recorded and was taken as a measure of depression.

\section{Collection of blood and serum samples:-}

Animals fasted overnight, then, all rats were anaesthetized by i.p injection of pentobarbital $(50 \mathrm{mg} / \mathrm{kg})(\mathrm{Samson}$ et al., 1957) and blood samples were obtained by cervical dislocation. Blood samples were collected in clean test tubes and centrifuged at $3000 \mathrm{rpm}$ for 15 minutes and the separated sera were then transferred into clean cuvette tube stored at $-80^{\circ} \mathrm{C}$ and used for the following measurements: Fasting glucose level by colorimetric assay (Henry, 1966). Serum insulin level using ELISA (Steiner \& Oyer, 1967), serum adiponectin level using ELISA (Yokota et al., 2000)and insulin resistance (HOMA IR \%) using HOMA calculator (version 2.2) (Manley et al., 2008).

\section{Tissue sampling:-}

After animals were sacrificed, perfuse brain tissue of each rat with a phosphate buffer saline (PBS) solution with pH 7.5 containing $0.16 \mathrm{mg} / \mathrm{ml}$ heparin to remove any red blood cells and clots. Each brain was carefully dissected out, 
weighed and homogenized in the suitable buffer. Then, supernatant were used for measurement of malondialdehyde (MDA) level by colorimetric assay (Ohkawa et al., 1979), catalase (CAT) level by colorimetric assay (Aebi, 1984), tumor necrosis factor alpha (TNF- $\alpha$ ) level using ELISA (Brouckaert et al., 1993), brain derived neurotropic factor (BDNF) level by ELISA (Baker-Herman et al., 2004) and serotonin (5-HT) level fluorometric assay based on the method described by (Jacobowitz \& Richardson, 1978).

Then, the sacrificed animals were packed in a special package according to safety precautions and infection control measures.

\section{Statistical analysis:-}

Data were expressed as mean \pm S.D. for the number of indicated animals. Statistical significance between means was analyzed by using One-way analysis of variance (ANOVA), followed by Tukey's post hoc test and $p$ values less than 0.05 were considered statistically significant. The analysis was performed by statistical package for the social science software (SPSS version 14.0) and GraphPad Prism software version 5.0 (San Diego, USA, 2007).

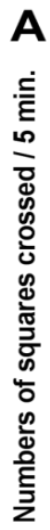

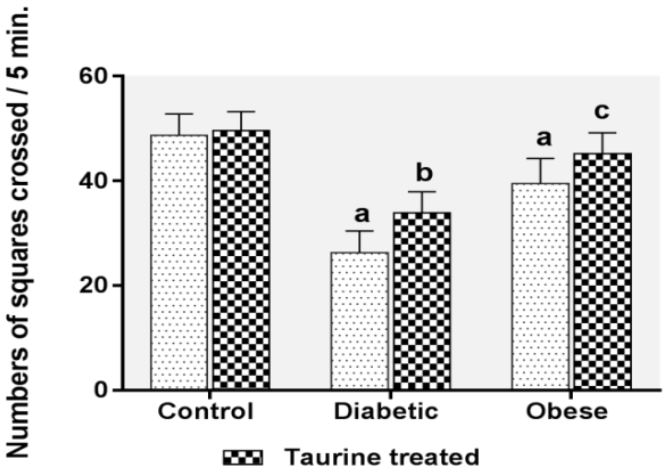

B

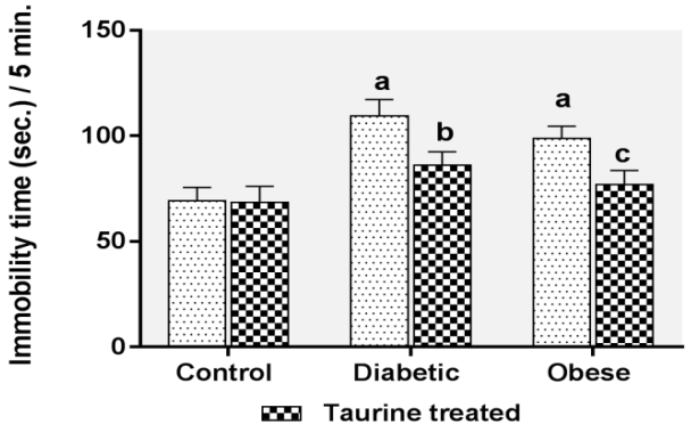

Fig. 1:-Effect of taurine on (A) number of squares crossed / $5 \mathrm{~min}$. in open field test and (B) immobility time (sec.) / $5 \mathrm{~min}$. of forced swim among studied groups: Values are expressed as means $\pm \mathrm{SD}\left(\mathrm{n}=10\right.$ per group); ${ }^{\mathrm{a}, \mathrm{b}, \mathrm{c}}$ denote a statistically significant at $\mathrm{p} \leq 0.05,{ }^{\mathrm{a}}$ denotes statistical significance when compared to control group, ${ }^{\mathrm{b}}$ denotes statistical significance when compared to diabetic group, ${ }^{\mathrm{c}}$ denotes statistical significance when compared to obese group using One-way ANOVA followed by Tukey's post-hoc test.

A

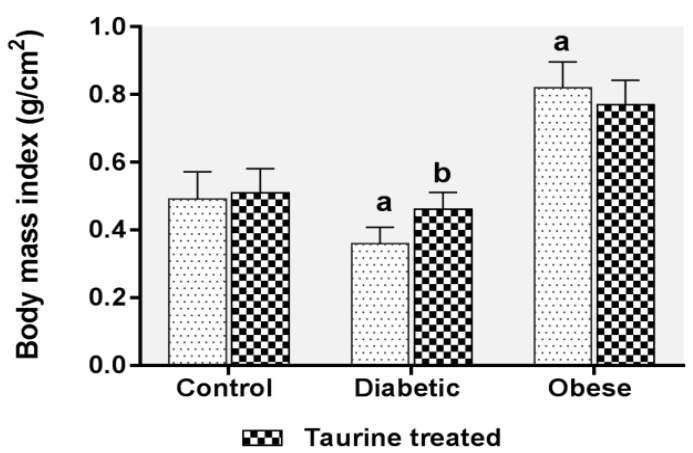

B

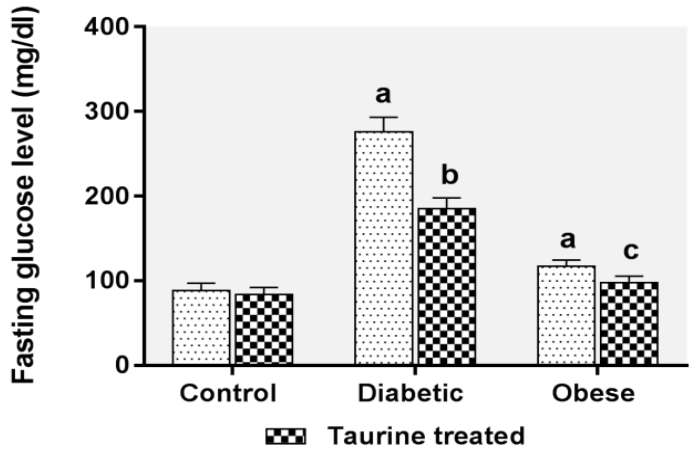

Fig. 2:-Effect of taurine on (A) body mass index $\left(\mathrm{g} / \mathrm{cm}^{2}\right)$ and (B) fasting glucose level $(\mathrm{mg} / \mathrm{dl})$ among studied groups: Values are expressed as means $\pm \mathrm{SD}$ ( $\mathrm{n}=10$ per group); ${ }^{\mathrm{a}, \mathrm{b}, \mathrm{c}}$ denote a statistically significant at $\mathrm{p} \leq 0.05$, ${ }^{\mathrm{a}}$ denotes statistical significance when compared to control group, ${ }^{b}$ denotes statistical significance when compared to diabetic group, ${ }^{\mathrm{c}}$ denotes statistical significance when compared to obese group using One-way ANOVA followed by Tukey's post-hoc test. 
A

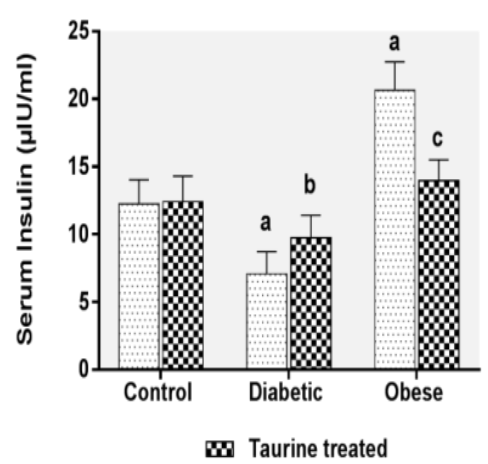

B

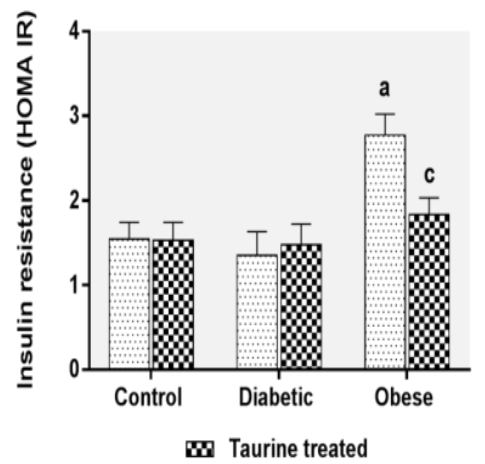

C

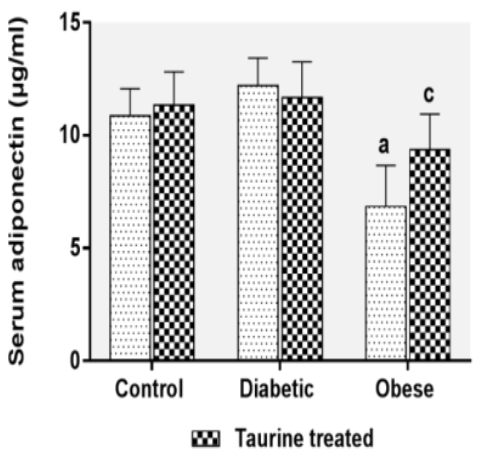

Fig. 3:-Effect of taurine on (A) serum insulin level ( $\mu \mathrm{IU} / \mathrm{ml}$ ), (B) HOMA IR and (C) serum adiponectin level $(\mathrm{ng} / \mathrm{ml})$ among studied groups: Values are expressed as means $\pm \mathrm{SD}\left(\mathrm{n}=10\right.$ per group); ${ }^{\mathrm{a}, \mathrm{b}, \mathrm{c}}$ denote a statistically significant at $\mathrm{p} \leq 0.05,{ }^{\mathrm{a}}$ denotes statistical significance when compared to control group, ${ }^{\mathrm{b}}$ denotes statistical significance when compared to diabetic group, ${ }^{c}$ denotes statistical significance when compared to obese group using One-way ANOVA followed by Tukey's post-hoc test.

A

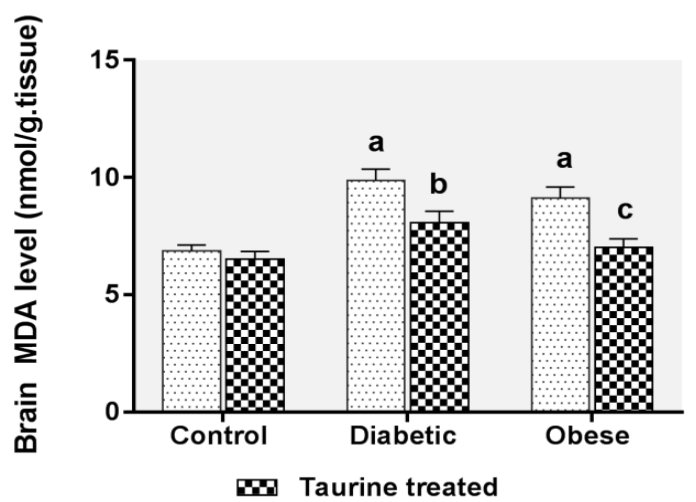

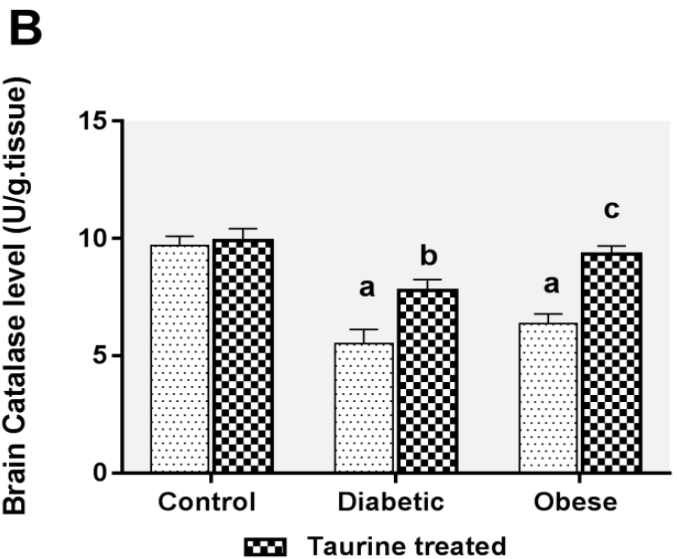

Fig. 4:-Effect of taurine on (A) brain malondialdehyde (MDA) (nmol/g.tissue) and (B) catalase levels (U/g.tissue) among studied groups: Values are expressed as means $\pm \mathrm{SD}\left(\mathrm{n}=10\right.$ per group); ${ }^{\mathrm{a}, \mathrm{b}, \mathrm{c}}$ denote a statistically significant at $\mathrm{p}$ $\leq 0.05,{ }^{\text {a }}$ denotes statistical significance when compared to control group, ${ }^{\mathrm{b}}$ denotes statistical significance when compared to diabetic group, ${ }^{\mathrm{c}}$ denotes statistical significance when compared to obese group using One-way ANOVA followed by Tukey's post-hoc test. 


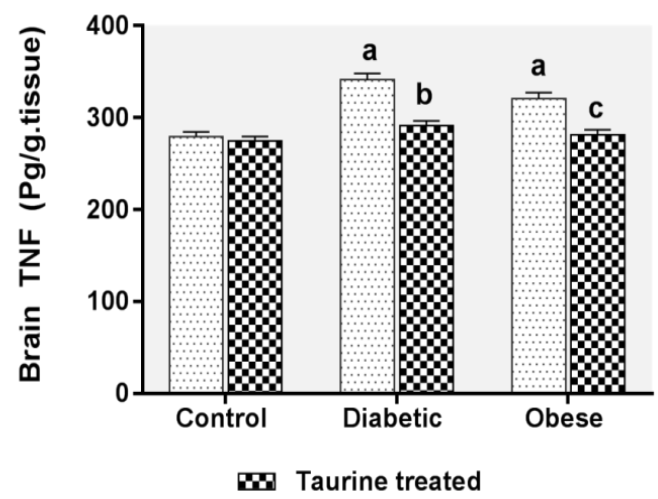

Fig. 5:-Effect of taurine on brain tumor necrosis factor alpha level (TNF- $\alpha$ ) (pg/ g.tissue) among studied groups: Values are expressed as means $\pm \mathrm{SD}$ ( $\mathrm{n}=10$ per group); ${ }^{\mathrm{a}, \mathrm{b}, \mathrm{c}}$ denote a statistically significant at $\mathrm{p} \leq 0.05,{ }^{\mathrm{a}}$ denotes statistical significance when compared to control group, ${ }^{\mathrm{b}}$ denotes statistical significance when compared to diabetic group, ${ }^{c}$ denotes statistical significance when compared to obese group using One-way ANOVA followed by Tukey's post-hoc test.

A

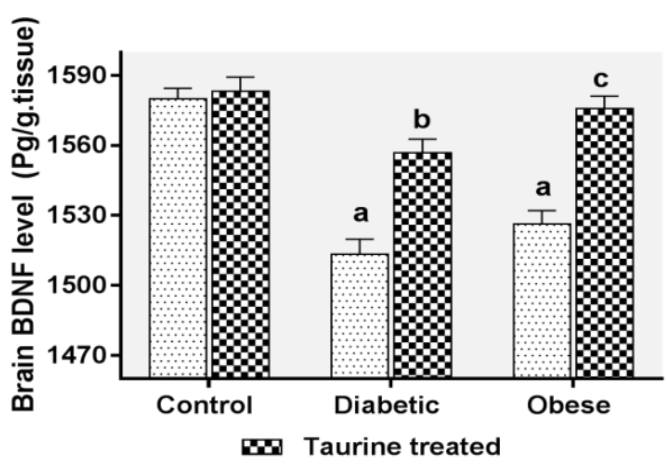

B

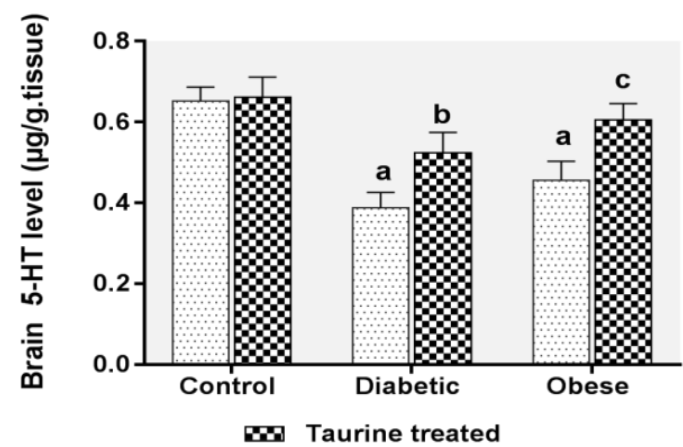

Fig. 6:-Effect of taurine on (A) brain derived neurotropic factor level (BDNF) (pg/g.tissue) and (B) serotonin level $(5-\mathrm{HT})\left(\mu \mathrm{g} / \mathrm{g}\right.$ tissue) among studied groups: Values are expressed as means $\pm \mathrm{SD}\left(\mathrm{n}=10\right.$ per group); ${ }^{\mathrm{a}, \mathrm{b}, \mathrm{c}}$ denote a statistically significant at $\mathrm{p} \leq 0.05,{ }^{\mathrm{a}}$ denotes statistical significance when compared to control group, ${ }^{\mathrm{b}}$ denotes statistical significance when compared to diabetic group, ${ }^{\mathrm{c}}$ denotes statistical significance when compared to obese group using One-way ANOVA followed by Tukey's post-hoc test.

\section{Results:-}

Effect of taurine on behavioural tests for diabetic and obese rats:-

As shown in Fig. $1 \mathrm{~A}$ and B, the number of squares crossed in OFT showed significant decrease associated with significant increase of the immobility time in FST in both diabetic and obese groups when compared to control group. Taurine administration significantly increases the number of crossed squares in OFT with significant decrease of the immobility time in FST when compared to diabetic and obese groups respectively.

Effect of taurine on fasting glucose and BMI of diabetic and obese rats:-

As shown in Fig. 2 A and B, levels of fasting blood glucose were significantly increased in both diabetic and obese groups when compared to control group. BMI was significantly decreased in diabetic rats, but significantly increased in obese ones compared to control group. Administration of taurine significantly decreased fasting glucose level with significant increased BMI compared to diabetic group. Also, taurine resulted in significant decrease of fasting glucose level without significant change in BMI compared to obese group. 
Effect of taurine on insulin, HOMA IR and adiponectin in diabetic and obese rats:-

As shown in Fig. 3 A, B and C, when compared to control group, there is significant decrease of serum insulin level without significant changes in insulin resistance or serum adiponectin level in diabetic group. However, serum insulin level and insulin resistance were significantly increased with significant decrease of serum adiponectin level in obese group. Administration of taurine to diabetic rats significantly increased serum insulin level without any significant changes in insulin resistance or serum adiponectin level as compared to diabetic group. In taurine treated obese group, there is significant decrease in serum insulin level and insulin resistance with significant increase in serum adiponectin level as compared to obese group.

\section{Effect of taurine on the oxidant/antioxidant status in the brain of diabetic and obese rats:-}

As shown in Fig. $4 \mathrm{~A}$ and B, MDA level in the brain showed significant increase associated with significant decrease of brain CAT level in both diabetic and obese groups compared to control group. However, taurine administration to diabetic and obese rats significantly decreased MDA level with significant increase of CAT level in the brain when compared to diabetic and obese groups respectively.

Effect of taurine on TNF- $\alpha$ in the brain of diabetic and obese rats:-

As shown in Fig. 5, TNF- $\alpha$ level in the brain significantly increased in both diabetic and obese groups as compared to control group. However, taurine administration to diabetic and obese rats significantly decreased brain TNF- $\alpha$ level when compared to diabetic and obese groups respectively.

Effect of taurine on serotonin and BDNF in the brain of diabetic and obese rats:-

As shown in Fig. 6 A and B, levels of serotonin and BDNF in the brain showed significant decrease in both diabetic and obese groups as compared to control group. Interestingly, taurine administration to diabetic and obese rats significantly increased brain serotonin and BDNF levels when compared to diabetic and obese groups respectively.

\section{Discussion:-}

Both diabetes and obesity exhibited depressive like behaviours manifested by significant increase in the frequency of passive (immobility) behaviours during the forced swim test (FST) (Castagne et al., 2011) associated with significant reduction in the number of crossings in the open field test (OFT) (Xia et al., 2016). There is also significant decrease in brain serotonin (5-HT) and BDNF levels in both diabetic and obese rats compared with the control values.

5-HT is a key neurotransmitter in the regulation of mood and it has been widely studied in depression pathophysiology (Papazoglou et al., 2015). Reduced brain 5-HT activity explain the behavioural despair revealed in the form of increased immobility time in FST (Santiago et al., 2010). Morever, there is a significant correlation between 5-HT plasma levels and behavioural changes obtained in OFT (Xia et al., 2016).

Brain derived neurotropic factor BDNF plays an important role in the neurogenesis and neuronal circuits survival and its reduction in various brain areas is a biological marker for depression (Autry \& Monteggia, 2012).

Most complications of DM as depression is attributed mainly to its accompanying hyperglycemia (Gaspar et al., 2016). Also, Bajaj et al. (2012) concluded that the presence of depression is correlated with the level of hyperglycemia.

Systemic IR in obesity is closely related to central IR of the brain which can manifest functionally as an impairment of cognition and mood (Arnold et al., 2018) and contributes to the onset and progression of depressive disorders (Detka et al., 2013).

Adiponectin has anti-inflammatory action and reduces TNF- $\alpha$ secretion (Esfahani et al., 2015).There is a negative correlation between obesity and circulating adiponectin especially with increased insulin resistance (IR) ( Ye \& Scherer, 2013). Thus, Chronic inflammation and reduced adiponectin in adipose tissue contribute to pathogenesis of IR, which links obesity to many complications (Aguilar-Valles et al., 2015).

There is loss of suppressive effect of insulin on MAO gene expression which increases the level of monoamine oxidases A and B (MAO A and B) resulting in increased serotonin clearance (Kleinridders et al., 2015). In addition, 
serotonin transporter (SERT) which is responsible for reuptake of serotonin is reduced in insulin resistant brain (Versteeg et al., 2017).

Oxidative stress (OS) resembles a hallmark of STZ-induced diabetes since it can promote reactive oxygen species (ROS) formation in various organs (Pandya et al., 2010). On the other hand, high fat diet (HFD) that increases fat mediated oxidative stress and decreases antioxidative enzyme activity (Yang et al., 2008). Pathways for reactive oxygen species (ROS) production are upregulated in both the liver and adipose tissue even before the onset of insulin resistance (Matsuzawa-Nagata et al., 2008). OS suppresses mRNA expression and secretion of adiponectin. So, markers of systemic oxidative stress correlate negatively with plasma adiponectin levels (Furukawa et al., 2017).

OS may contribute to the pathogenesis of depression by interfering with neurotransmission and neuroplasticity (Bajpai et al., 2014). It is associated with increased activity of monoamine oxidase enzyme that is involved in oxidative deamination of serotonin. In addition, lipid peroxidation reduces stability of membranes, activity of ion channels, affects neurotransmitters release and 5-HT signaling process through MDA formation (Vavakova et al., 2015).

OS reduces cAMP response element binding (CREB) protein expression leading to a decrement in BDNF gene expression and content (Kapczinski et al., 2008).

Diabetes is an inflammatory disease since hyperglycemia induced oxidative stress activates inflammatory signaling pathways and increases production of several inflammatory cytokines(Shi et al., 2011). Oxidative stress and inflammation is toxic to the CNS which impairs neuronal transmission (Caletti et al., 2017).

Increased production of pro-inflammatory cytokines affects the metabolism of serotonin by activation of indolamine-2, 3-dioxygenase (IDO) that metabolizes tryptophan into kynurenine (KYN) (Myint et al., 2012).IDO activation shifts tryptophan metabolism from the serotonin towards the KYN pathway which may explain the low availability of serotonin in depression(Ma et al., 2016).

Inflammation reduces the expression of neurotrophins and influences BDNF tyrosine receptor kinase B (TrkB) phosphorylation, thereby further interfering with BDNF signaling (Felger \& Lotrich, 2013). In addition, large amounts of pro-inflammatory cytokines reduce 5-HT receptor dependent cyclic AMP response element-binding (CREB) activation, which in turn decreases BDNF gene expression and level. Thus, a strong connection has been established between BDNF, depression and serotonergic neurotransmission (Haase \& Brown, 2014).

Hence, multiple mechanisms are related to reduction of brain serotonin and BDNF levels in diabetes and HF diet induced obesity such as central insulin resistance, development of oxidative stress and neuroinflammation.

However, the results of the present work showed that taurine has an antidepressant effect as evidenced by the significant decrease in immobility behaviours in the FST and significant increase in the number of crossings in diabetic and obese rats. These findings are in agreement withToyoda \& Iio (2013) who reported that taurine has antidepressant like effects and an ability to change depression related signaling cascades in the hippocampus in rats. In contrast, Whirley \& Einat (2008) did not find any antidepressant effect of taurine after three consecutive days of intraperitoneal administration in on obese mice.

The improvement of depressive like behaviours in taurine treated diabetic rats may be due to its hypoglycemic effect revealed in our results. It increases insulin release from pancreatic beta cells as it is taken up by the taurine transporter (TauT) on B cells and inactivates the ATP-sensitive K+-channels leading to opening of voltage-gated calcium channels with increase calcium concentration resulting in subsequent release of insulin (Vettorazzi et al., 2014).

The beneficial effect of taurine against insulin resistance may be owing to its antioxidant and anti inflammatory effects. Also, it has the ability to increase insulin signaling since it modulates the insulin signal transduction pathways by inhibiting the cellular protein tyrosine phosphatase (PTP) activity in order to increase tyrosine phosphorylation (Nandhini et al., 2005). 
Increased adiponectin level in the present study is associated with improvement of insulin sensitivity as evidenced by decreased HOMA-IR values. This owes directly to increased adiponectin synthesis by taurine or due to improvement of other factors including glucose metabolism ( Murakami, 2015).

This study showed that taurine has strong antioxidant and anti inflammatory effects as it significantly decreased brain MDA and brain TNF- $\alpha$ level and elicited a significant increase in brain catalase activity.

The antioxidant effects of taurine have been attributed to its ability to stabilize the biomembranes, to reduce the peroxidation of unsaturated membrane lipids and to scavenge reactive oxygen species (ROS) (Ikubo et al., 2011). However, Schaffer et al. (2009) reported that taurine by itself cannot directly scavenge classical ROS, but it is capable of inhibiting ROS generation ( La Puerta et al., 2010).

Taurine can also function as an indirect antioxidant via its regulatory effect on mitochondrial functions preserving activity of the electron transport chain and integrity of its complexes, maintaining a normal flow of electrons and preventing their deviation to generate of ROS (Jong et al., 2012).

Anti-inflammatory action of taurine is related to its antioxidant ability as it can react with hypochlorous acid produced by activated leukocytes to form taurine chloramine (Tau-Cl), which plays a direct antioxidant role as well as an anti inflammatory effect with inhibiton of TNF-aexpression and secretion (Chen et al., 2016).

Accordingly, increased brain serotonin level in taurine treated groups may be due to improving insulin sensitivity, antioxidant and anti-inflammatory effects of taurine. In addition, taurine can increase expression of tryptophan hydroxylase (TPH) which is the rate limiting enzyme for the synthesis of serotonin (Liu et al., 2013).

The antioxidant and anti-inflammatory activities of taurine may also contribute to its neuroprotective effect in this study through increasing level of brain BDNF.

Therefore, taurine can increase brain serotonin and BDNF levels through its hypoglycemic effect, its ability to reduce insulin resistance, its positive correlation with adiponectin level, antioxidant and anti inflammatory actions as well.

\section{Conclusion:-}

We conclude that antidepressant like effect of taurine is due to its hyporglycemic effect with improvement of insulin sensitivity. Morever, it seems to be related to amelioration of oxidative stress and neuroinflammation.

\section{Recommendation:-}

Thus, our data suggest that taurine may be used as an adjuvant therapy with other traditional antidepressant drugs in diabetic and obese patients suffering from depression. It can increase the therapeutic effect and tolerance to these drugs and decrease risk from their side effects.

\section{References:-}

1. Aebi H.B. in E. (1984) : Catalase in vitro. inOxyg. Radicals Biol. Syst., 121-126.

2. Aguilar-Valles A., Inoue W., Rummel C. and Luheshi G.N. (2015) : Obesity, adipokines and neuroinflammation. Neuropharmacology, 96, 124-134.

3. Arnold S.E., Arvanitakis Z., Macauley-Rambach S.L., Koenig A.M., Wang H.-Y., Ahima R.S., et al. (2018) :Brain insulin resistance in type 2 diabetes and Alzheimer disease: concepts and conundrums. Nat. Rev. Neurol., 14(3), 168-181.

4. Autry A.E. and Monteggia L.M. (2012) : Brain-Derived Neurotrophic Factor and Neuropsychiatric Disorders. Pharmacol. Rev., 64(2), 238- 258.

5. Bajaj S., Agarwal S.K., Varma A. and Singh V.K. (2012) : Association of depression and its relation with complications in newly diagnosed type 2 diabetes. Indian J. Endocrinol. Metab., 16(5), 759-763.

6. Bajpai A., Verma A.K., Srivastava M. and Srivastava R. (2014) : Oxidative Stress and Major Depression. J. Clin. Diagn. Res., 8(12), CC04-CC07. 
7. Baker-Herman T.L., Fuller D.D., Bavis R.W., Zabka A.G., Golder F.J., Doperalski N.J., et al. (2004): BDNF is necessary and sufficient for spinal respiratory plasticity following intermittent hypoxia. Nat. Neurosci., 7(1), 48-55.

8. Brouckaert P., Libert C., Everaerdt B., Takahashi N., Cauwels A. and Fiers W. (1993) :Tumor necrosis factor, its receptors and the connection with interleukin 1 and interleukin 6. Immunobiology, 187(3-5), 317-329.

9. Caletti G., Almeida F.B., Agnes G., Nin M.S., Barros H.M. and Gomez R. (2015) :Antidepressant dose of taurine increases mRNA expression of GABAA receptor alpha2 subunit and BDNF in the hippocampus of diabetic rats. Behav. Brain Res., 283, 11-15.

10. Caletti G., Herrmann A.P., Pulcinelli R.R., Steffens L., Moras A.M., Vianna P., et al. (2017) :Taurine counteracts the neurotoxic effects of streptozotocin-induced diabetes in rats. Amino Acids, 50(1):95-104.

11. Castagne V., Moser P., Roux S. and Porsolt R.D. (2011) :Rodent models of depression: forced swim and tail suspension behavioral despair tests in rats and mice. Curr. Protoc. Neurosci., Chapter 8, Unit 8.10A, 1-14.

12. Chen W., Guo J., Zhang Y. and Zhang J. (2016) :The beneficial effects of taurine in preventing metabolic syndrome. Food Funct., 7(4), 1849-1863.

13. Detka J., Kurek A., Basta-Kaim A., Kubera M., Lasoñz W. and Budziszewska B. (2013) :Neuroendocrine link between stress, depression and diabetes. Pharmacol. Reports, 65(6), 1591-1600.

14. Esfahani M., Movahedian A., Baranchi M. and Goodarzi M.T. (2015) :Adiponectin: an adipokine with protective features against metabolic syndrome. Iran. J. Basic Med. Sci., 18(5), 430-442.

15. Felger J.C. and Lotrich F.E. (2013) :Inflammatory cytokines in depression: neurobiological mechanisms and therapeutic implications. Neuroscience, 246, 199-229.

16. Furukawa S., Fujita T., Shimabukuro M., Iwaki M., Yamada Y., Nakajima Y., et al. (2017) : Increased oxidative stress in obesity and its impact on metabolic syndrome. J. Clin. Invest., 114(12), 1752-1761.

17. Gaspar J.M., Baptista F.I., Macedo M.P. and Ambrósio A.F. (2016) :Inside the Diabetic Brain: Role of Different Players Involved in Cognitive Decline. ACS Chem. Neurosci., 7(2), 131-142.

18. Haase J. and Brown E. (2014) :Integrating the monoamine, neurotrophin and cytokine hypotheses of depression - a central role for the serotonin transporter?. Pharmacol. Ther., 147, 1-11.

19. Henry R.J. (1966) : Clinical chemistry : principles and techniques. J Clin. Pathol., 19(2): 205.

20. Ikubo N., Saito M., Tsounapi P., Dimitriadis F., Ohmasa F., Inoue S., et al. (2011) :Protective effect of taurine on diabetic rat endothelial dysfunction. Biomed. Res., 32(3), 187-193.

21. Jacobowitz D.M. and Richardson J.S. (1978) :Method for the rapid determination of norepinephrine, dopamine, and serotonin in the same brain region. Pharmacol. Biochem. Behav., 8(5), 515-519.

22. Jong C.J., Azuma J. and Schaffer S. (2012) :Mechanism underlying the antioxidant activity of taurine: prevention of mitochondrial oxidant production. Amino Acids, 42(6), 2223-2232.

23. Kapczinski F., Frey B.N., Andreazza A.C., Kauer-Sant'Anna M., Cunha A.B.M. and Post R.M. (2008) :Increased oxidative stress as a mechanism for decreased BDNF levels in acute manic episodes. Rev. Bras. Psiquiatr., 30(3), 243-245.

24. Kearns B., Rafia R., Leaviss J., Preston L., Brazier J.E., Palmer S., et al. (2017) :The cost-effectiveness of changes to the care pathway used to identify depression and provide treatment amongst people with diabetes in England: a model-based economic evaluation. BMC Health Serv. Res., 17(78), 1-10.

25. Kim H.K., Nunes P.V., Oliveira K.C., Young L.T. and Lafer B. (2016) :Neuropathological relationship between major depression and dementia: A hypothetical model and review. Prog. Neuro-Psychopharmacology Biol. Psychiatry, 67, 51-57.

26. Kleinridders A., Cai W., Cappellucci L., Ghazarian A., Collins W.R., Vienberg S.G., et al. (2015) : Insulin resistance in brain alters dopamine turnover and causes behavioral disorders. Proc. Natl. Acad. Sci. U. S. A., 112(11), 3463-3468.

27. De la Puerta C., Arrieta F.J., Balsa J.A., Botella-Carretero J.I., Zamarron I. and Vazquez C. (2010):Taurine and glucose metabolism: a review. Nutr. Hosp., 25(6), 910-919.

28. Lang U.E. and Borgwardt S. (2013) :Molecular Mechanisms of Depression: Perspectives on New Treatment Strategies. Cell. Physiol. Biochem., 31(6), 761-777.

29. Liu X., Piao F. and Li Y. (2013) :Protective effect of taurine on the decreased biogenic amine neurotransmitter levels in the brain of mice exposed to arsenic. Adv. Exp. Med. Biol., 776, 277-287.

30. Ma K., Zhang H. and Baloch Z. (2016) :Pathogenetic and therapeutic applications of tumor necrosis factor- $\alpha$ (TNF- $\alpha$ ) in major depressive disorder: A systematic review. Int. J. Mol. Sci., 17(5), 1-21.

31. Manley S.E., Luzio S.D., Stratton I.M., Wallace T.M. and Clark P.M.S. (2008) :Preanalytical, analytical, and computational factors affect homeostasis model assessment estimates. Diabetes Care, 31(9), 1877-1883. 
32. Matsuzawa-Nagata N., Takamura T., Ando H., Nakamura S., Kurita S., Misu H., et al. (2008) : Increased oxidative stress precedes the onset of high-fat diet-induced insulin resistance and obesity. Metabolism., 57(8), 1071-1077.

33. Menzie J., Pan C., Prentice H. and Wu J.-Y. (2014) :Taurine and central nervous system disorders. Amino Acids, 46(1), 31-46.

34. deMorais H., de Souza C.P., da Silva L.M., Ferreira D.M., Werner M.F., Andreatini R., et al. (2014):Increased oxidative stress in prefrontal cortex and hippocampus is related to depressive-like behavior in streptozotocindiabetic rats. Behav. Brain Res., 258, 52-64.

35. Murakami S. (2015) :Role of taurine in the pathogenesis of obesity. Mol. Nutr. Food Res., 59(7), $1353-1363$.

36. Myint A.M., Schwarz M.J. and Müller N. (2012) :The role of the kynurenine metabolism in major depression. J. Neural Transm., 119(2), 245-251.

37. Nandhini A.T., Thirunavukkarasu V. and Anuradha C. V (2005) :Taurine modifies insulin signaling enzymes in the fructose-fed insulin resistant rats. Diabetes Metab., 31(4), 337-344.

38. Novelli E.L., Diniz Y.S., Galhardi C.M., Ebaid G.M., Rodrigues H.G., Mani F., et al. (2007) : Anthropometrical parameters and markers of obesity in rats. Lab. Anim., 41(1), 111-119.

39. Ohkawa H., Ohishi N. and Yagi K. (1979) :Assay for lipid peroxides in animal tissues by thiobarbituric acid reaction. Anal. Biochem., 95(2), 351-358.

40. Otte C., Gold S.M., Penninx B.W., Pariante C.M., Etkin A., Fava M., et al. (2016) : Major depressive disorder. Nat. Rev. Dis. Prim., 2, 1-20.

41. Pandya K.G., Patel M.R. and Lau-Cam C.A. (2010) :Comparative study of the binding characteristics to and inhibitory potencies towards PARP and in vivo antidiabetogenic potencies of taurine, 3-aminobenzamide and nicotinamide. J. Biomed. Sci., 17 (Suppl 1), 1-15.

42. Papazoglou I.K., Jean A., Gertler A., Taouis M. and Vacher C.M. (2015) :Hippocampal GSK3 $\beta$ as a Molecular Link Between Obesity and Depression. Mol. Neurobiol., 52(1), 363-374.

43. Patke A., Tripathi R., Patke V.G., Sonawane D. and Rege N. (2015) :Antidepressant activity of Simvastatin in behavioral models of depression in rats. Int. J. Res. Med. Sci., 3(7), 1666-1671.

44. Porsolt R.D., Anton G., Blavet N. and Jalfre M. (1978) :Behavioural despair in rats: a new model sensitive to antidepressant treatments. Eur. J. Pharmacol., 47(4), 379-391.

45. Quinn C.C., Swasey K.K., Crabbe J.C., Shardell M.D., Terrin M.L., Barr E.A., et al. (2017) :The Impact of a Mobile Diabetes Health Intervention on Diabetes Distress and Depression Among Adults: Secondary Analysis of a Cluster Randomized Controlled Trial. JMIR mHealthuHealth, 5(12), 1-27.

46. Ramos A., Berton O., Mormède P. and Chaouloff F. (1997) :A multiple-test study of anxiety-related behaviours in six inbred rat strains. Behav. Brain Res., 85(1), 57-69.

47. Redivo D.D., Schreiber A.K., Adami E.R., Ribeiro D.E., Joca S.R.L., Zanoveli J.M., et al. (2016) : Effect of omega-3 polyunsaturated fatty acid treatment over mechanical allodynia and depressive-like behavior associated with experimental diabetes. Behav. Brain Res., 298, 57-64.

48. Salze G.P. and Davis D.A. (2015) :Taurine: a critical nutrient for future fish feeds. Aquaculture, 437, $215-229$.

49. Samson F., Colip F. \& Patterson J. (1957): Procedure for the Use of Sodium Pentobarbital (Nembutal $\left.{ }^{\circledR}\right)$ Anesthesia in Classroom Experiments with Rats. Transactions of the Kansas Academy of Science, (1903), 425-428

50. Santiago R.M., Barbieiro J., Lima M.M.S., Dombrowski P.A., Andreatini R. and Vital M.A.B.F. (2010) :Depressive-like behaviors alterations induced by intranigral MPTP, 6-OHDA, LPS and rotenone models of Parkinson's disease are predominantly associated with serotonin and dopamine. Prog. Neuropsychopharmacol. Biol. Psychiatry, 34(6), 1104-1114.

51. Sarris J. (2017) :Clinical use of nutraceuticals in the adjunctive treatment of depression in mood disorders. Australas. Psychiatry, 25(4), 369-372.

52. Schaffer S.W., Azuma J. and Mozaffari M. (2009) :Role of antioxidant activity of taurine in diabetes. Can. J. Physiol. Pharmacol., 87(2), 91-99.

53. Schuch F.B., Deslandes A.C., Stubbs B., Gosmann N.P., Silva C.T.B. da and Fleck M.P. de A. (2016) :Neurobiological effects of exercise on major depressive disorder: A systematic review. Neurosci. Biobehav. Rev., 61, 1-11.

54. Sharma S. and Fulton S. (2013) :Diet-induced obesity promotes depressive-like behaviour that is associated with neural adaptations in brain reward circuitry. Int. J. Obes., 37(3), 382-389.

55. Shi Y.C., Liao J.W. and Pan T.M. (2011) :Antihypertriglyceridemia and anti-inflammatory activities of monascus-fermented dioscorea in streptozotocin-induced diabetic rats. Exp. Diabetes Res., 2011(ID 710635),111. 
56. Steiner D.F. and Oyer P.E. (1967) :The biosynthesis of insulin and a probable precursor of insulin by a human islet cell adenoma. Proc. Natl. Acad. Sci. U. S. A., 57(2), 473-480.

57. Sweeney P., O'Hara K., Xu Z. and Yang Y. (2017) :HFD-induced energy states-dependent bidirectional control of anxiety levels in mice. Int. J. Obes. (Lond)., 41(8), 1237-1245.

58. Toyoda A. and Iio W. (2013) :Antidepressant-like effect of chronic taurine administration and its hippocampal signal transduction in rats. Adv. Exp. Med. Biol., 775, 29-43.

59. Vavakova M., Durackova Z. and Trebaticka J. (2015) :Markers of Oxidative Stress and Neuroprogression in Depression Disorder. Oxid. Med. Cell. Longev., 2015(ID 898393),1-12.

60. Versteeg R.I., Koopman K.E., Booij J., Ackermans M.T., Unmehopa U.A., Fliers E., et al. (2017) :Serotonin Transporter Binding in the Diencephalon Is Reduced in Insulin-Resistant Obese Humans. Neuroendocrinology, $105(2), 141-149$.

61. Vettorazzi J.F., Ribeiro R.A., Santos-Silva J.C., Borck P.C., Batista T.M., Nardelli T.R., et al. (2014) :Taurine supplementation increases KATP channel protein content, improving $\mathrm{Ca} 2+$ handling and insulin secretion in islets from malnourished mice fed on a high-fat diet. Amino Acids, 46(9), 2123- 36.

62. Whirley B.K. and Einat H. (2008) :Taurine trials in animal models offer no support for anxiolytic, antidepressant or stimulant effects. Isr. J. Psychiatry Relat. Sci., 45(1), 11-18.

63. Wu G.F., Ren S., Tang R.-Y., Xu C., Zhou J.Q., Lin S.M., et al. (2017) : Antidepressant effect of taurine in chronic unpredictable mild stress-induced depressive rats. Sci. Rep., 7(4989),1-14.

64. Xia Z., Wei H., Duan J., Zhou T., Yang Z. and Xu F. (2016) :Chronic unpredicted mild stress-induced depression alter saxagliptin pharmacokinetics and CYP450 activity in GK rats. Peer J, 4, 1-15.

65. Yang R.L., li wu, Shi Y.H. and Le G. (2008) Lipoic acid prevents high-fat diet-induced dyslipidemia and oxidative stress: A microarray analysis Nutrition. Nutrition, 24(6), 582-588.

66. Ye R. and Scherer P.E. (2013) :Adiponectin, driver or passenger on the road to insulin sensitivity? Mol. Metab., 2(3), 133-141.

67. Yokota T., Oritani K., Takahashi I., Ishikawa J., Matsuyama A., Ouchi N., et al. (2000) :Adiponectin, a new member of the family of soluble defense collagens, negatively regulates the growth of myelomonocytic progenitors and the functions of macrophages. Blood, 96(5), 1723-1732.

68. Zanoveli J.M., Morais H., Dias I.C., Schreiber A.K., Souza C.P. and Cunha J.M. (2016) :Depression Associated with Diabetes: From Pathophysiology to Treatment. Curr. Diabetes Rev., 12(3), 165-178. 